УДК 621.315 .592

\title{
Исследование фотоотклика графена, полученного методом химического осаждения из газовой фазы
}

\author{
() А.В. Бабичев ${ }^{1}$, С.А. Кадинская ${ }^{1}$, К.Ю. Шубина ${ }^{1}$, А.А. Васильев ${ }^{1}$, А.А. Блохин ${ }^{2,3}$, \\ Э.И. Моисеев ${ }^{4}$, С.А. Блохин ${ }^{3}$, И.С. Мухин ${ }^{1,5}$, И.А. Елисеев ${ }^{3}$, В.Ю. Давыдов ${ }^{3}$, \\ П.Н. Брунков ${ }^{3}$, Н.В. Крыжановская ${ }^{4}$, А.Ю. Егоров 5
}

${ }^{1}$ Санкт-Петербургский национальный исследовательский Академический университет им. Ж.И. Алфёрова

Российской академии наук, 194021 Санкт-Петербург, Россия

${ }^{2}$ Научно-технологический центр микроэлектроники и субмикронных гетероструктур Российской академии наук, 194021 Санкт-Петербург, Россия

${ }^{3}$ Физико-технический институт им. А.Ф. Иоффре Российской академии наук, 194021 Санкт-Петербург, Россия

${ }^{4}$ Национальный исследовательский университет „Высшая школа экономики“, 190008 Санкт-Петербург, Россия

${ }^{5}$ Национальный исследовательский университет ИТМО,

197101 Санкт-Петербург, Россия

E-mail: a.babichev@mail.ioffe.ru

Поступила в Редакцию 6 мая 2020 г.

В окончательной редакции 12 мая 2020 г.

Принята к публикации 12 мая 2020 г.

\begin{abstract}
Представлены результаты экспериментов по созданию и изучению свойств фотодетекторных структур на основе монослойного графена, полученного методом химического осаждения из газовой фазы. В качестве базовой конструкции взята геометрия вертикального микрорезонатора $\mathrm{Ta}_{2} \mathrm{O}_{5}$ с нижним диэлектрическим распределенным брэгговским отражателем $\mathrm{SiO}_{2} / \mathrm{Ta}_{2} \mathrm{O}_{5}$ с резонансной длиной волны вблизи 850 нм. Проведена оптимизация условий переноса и формирования мез в слое графена на поверхности микрорезонатора. Диагностика кристаллического качества графена после формирования мез в слое графена и контактных площадок методом комбинационного рассеяния света свидетельствует о монослойности графена с низкой интенсивностью особенности в его спектре, отвечающей за дефектность структуры. Проведено измерение величины фототока при локальной оптической накачке.
\end{abstract}

Ключевые слова: фотодетекторные структуры, монослойный графен, микрорезонатор, меза.

DOI: 10.21883/FTP.2020.09.49813.9422

\section{1. Введение}

Наряду с активно ведущимися исследованиями фотодетекторов на основе соединений $\mathrm{A}^{\mathrm{III}} \mathrm{B}^{\mathrm{V}}[1,2]$ в последнее время наблюдается значительный интерес к новым типам фотодетекторов, в том числе на основе графена [3-23]. При учете толщины в $3.35 \AA$ графен имеет коэффициент поглощения, превышающий на 1-3 порядка значения для типичных полупроводников (InGaAs, GaAs, $\mathrm{Ge})$ [24]. Однако в силу малой длины взаимодействия графен поглощает лишь $2.3 \%$ от падающего излучения (соответствует видимой области спектра) [25]. В отличие от большинства других материалов, в графене отсутствует запрещенная зона, поэтому нет ограничения на длину волны поглощения (край поглощения). Как следствие, графен поглощает излучение от ультрафиолетовой до терагерцовой области спектра [12,26-28].

К настоящему времени предложено несколько подходов по повышению поглощения графена, основными из которых являются: использование плазмонных наноструктур [29], использование наночастиц [30], интеграция графена в волноводную конфигу- рацию [4,30-32], формирование легированных графеновых наноразмерных дисков [33], формирование сверхрешеток графен-диэлектрик [34], интеграция с фоточувствительными материалами (квантовые точки) [35], формирование резонансного фотодетектора [36-38]. Как показано в [38], за счет использования геометрии резонансного фотодетектора удается повысить поглощение графена вплоть до 100\%, что обусловлено локальным увеличением интенсивности поля в области поглощения (расположение в пучности электромагнитного поля стоячей волны микрорезонатора) [39], а также многопроходной геометрией света в микрорезонаторе. Более того, использование микрорезонатора позволяет достичь узкой спектральной селективности детектируемого излучения, что имеет важное значение для мультиплексирования c разделением по длинам волн (wavelength division multiplexing) [40].

В настоящей работе представлены результаты по формированию и исследованию оптических характеристик фотодетекторных структур на основе графена, полученного методом химического осаждения из газовой фазы. 


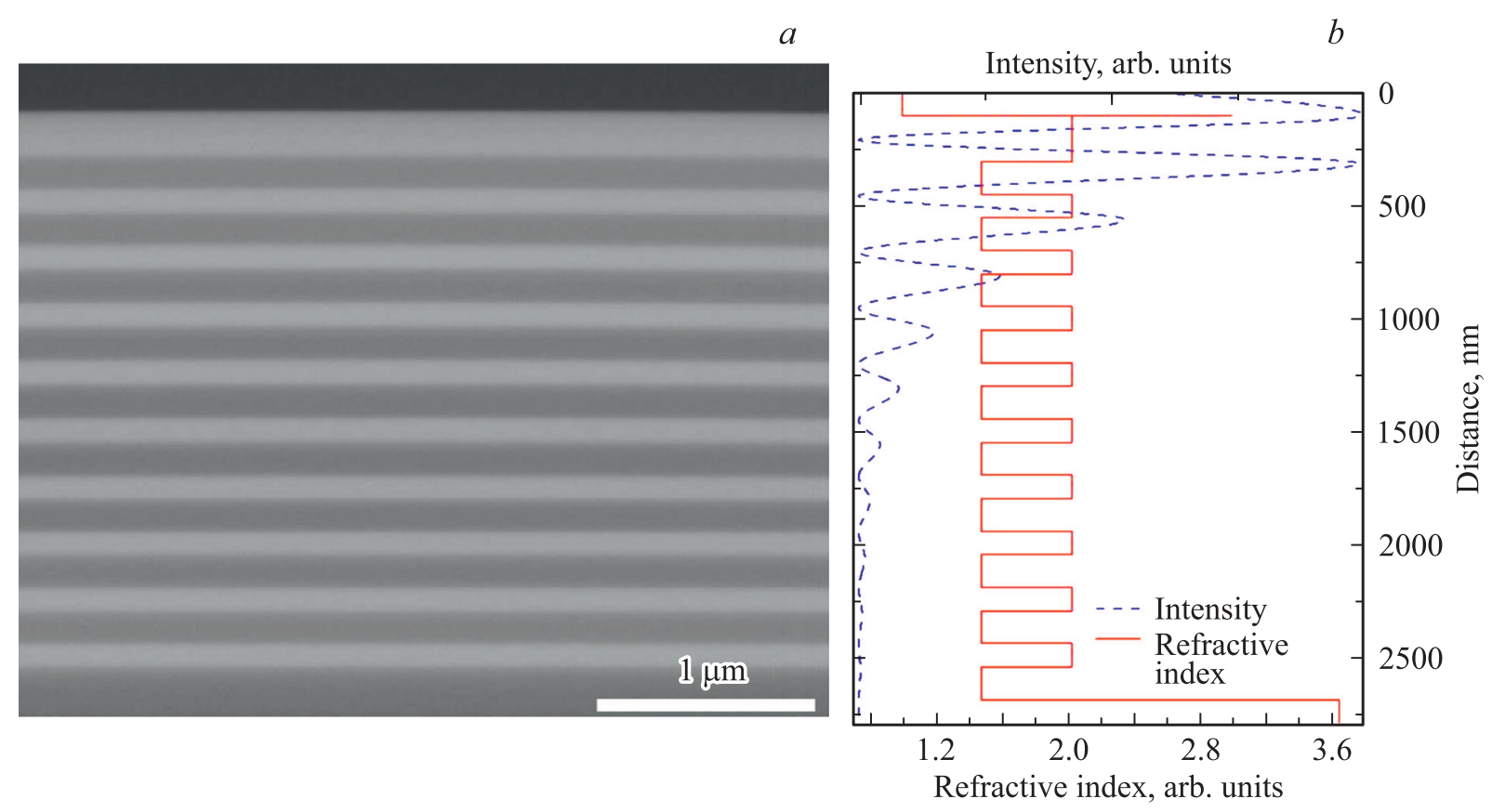

Рис. 1. $a-$ изображение диэлектрического микрорезонатора $\mathrm{SiO}_{2} / \mathrm{Ta}_{2} \mathrm{O}_{5}$ в геометрии поперечного сечения (110), полученное с помощью РЭМ; $b$ - распределение интенсивности электромагнитного поля в микрорезонаторе (штриховая линия), изменение показателя преломления в микрорезонаторе (сплошная линия).

\section{2. Эксперимент}

На рис. 1 приведено схематическое изображение диэлектрического микрорезонатора спектрального диапазона 850 нм, состоящего из диэлектрического распределенного брэгговского отражателя (РБО) на основе 10 пар четвертьволновых слоев $\mathrm{SiO}_{2} / \mathrm{Ta}_{2} \mathrm{O}_{5}$ и оптического резонатора $\mathrm{Ta}_{2} \mathrm{O}_{5}$ толщиной $\lambda / 2$. Использование микрорезонатора позволило увеличить интенсивность электромагнитного поля в области расположения графена в 4 раза. Структура диэлектрического микрорезонатора формировалась методом реактивного магнетронного распыления кварцевой мишени на переменном токе в атмосфере чистого аргона и танталовой мишени в газовой смеси из аргона и кислорода на постоянном токе при температуре $\sim 100^{\circ} \mathrm{C}$, что позволяет также использовать „взрывную“ (lift-off) технологию локального формирования диэлектрических отражателей $[41,42]$.

В качестве подложки использовался атомно-гладкий кремний (100) с целью повышения адгезии слоев диэлектрика к подложке при осаждении в режиме низких температур. Следует отметить, что большой контраст показателей преломления является несомненным преимуществом пары диэлектриков $\mathrm{SiO}_{2}-\mathrm{TiO}_{2}$, однако ее использование в рамках данной технологии реактивного магнетронного распыления имеет ряд трудностей, сопряженных с более высокой шероховатостью поверхности и оптической неоднородностью, которые обусловлены блочной структурой слоев $\mathrm{TiO}_{2}$ (наличием межфазных границ) [41].
После формирования диэлектрического микрорезонатора на поверхности образца методом взрывной литографии создавались метки совмещения на основе слоев металлизации $\mathrm{Ti} / \mathrm{Au}$ с толщинами 10 и 250 нм. При взрывной литографии использована схема на основе фоторезиста (ФР) AZ MIR 701 с подслоем Microchem PMGI SF7. Экспозиция ФP проводилась на установке лазерной литографии Heidelberg DWL 66FS. После формирования меток совмещения осуществлялся перенос монослойного графена, полученного методом химического осаждения из газовой фазы (метод CVD) через жидкость [43-48]. Перенос графена с исходной поверхности медной фольги осуществлялся за счет жидкостного травления меди в растворе персульфата аммония с предварительным нанесением слоя полиметилметакрилата (РММА) на поверхность графена [49-52]. После переноса графена слой РММА удалялся за счет кипячения образца в ацетоне/изопропаноле. Формирование мез в слое графена проводилось за счет создания маски на основе фоторезиста и последующего травления в кислородной плазме. При формировании маски использовался позитивный фоторезист AZ MIR 701. Травление мез в слое графена проведено на установке плазменной очистки Pink Plasma Finish V15-G. Поток кислорода составлял $60 \mathrm{~cm}^{3} /$ мин, мощность плазмы равнялась $400 \mathrm{BT}$, частота генератора 2.45 ГГц. После формирования мез в слое графена осуществлялось снятие фоторезиста за счет промывки образца в ацетоне и изопропаноле с последующим созданием маски под взрывную фотолитографию методом лазерной литографии. В качестве 
контактной металлизации использовался слой Тi/Au толщиной 10 и 20 нм.

Для анализа микрорельефа поверхности диэлектрического микрорезонатора $\mathrm{SiO}_{2} / \mathrm{Ta}_{2} \mathrm{O}_{5}$ были проведены исследования методом атомно-силовой микроскопии (ACM) на микроскопе Veeco Dimension 3100 с использованием контактного режима. Измерения проведены с использованием зондов RTESP с радиусом закругления 10 нм при комнатной температуре и влажности атмосферы $\sim 20 \%$.

Для измерения спектров отражения на макромасштабе использована установка измерения фотолюминесценции и оптического отражения Accent RPM Sigma.

Для диагностики кристаллического качества графена после формирования мез в слое графена и контактных площадок использован метод комбинационного рассеяния света (КРС). Измерения проводились на спектрометрической установке Horiba Jobin-Yvon T64000, укомплектованной конфокальным оптическим микроскопом, что позволяло получать информацию из области диаметром $\sim 1$ мкм. Для возбуждения при измерении спектров КРС использовался твердотельный Nd:YAG-лазер производства фирмы Torus (Великобритания) с длиной волны излучения $\lambda=532$ нм. Мощность лазерного излучения на образце составляла $2 \mathrm{MBT}$.

Для оценки проводимости мез графена, сформированных на поверхности микрорезонатора, применялся анализатор параметров полупроводниковых прибоpoв Agilent B1500, совмещенный с зондовой станцией Zuss PM6. Измерение проводимости проводилось двухзондовым методом.

Фототок измеряли при оптической накачке лазером в различных локальных областях мез в слое графена между контактными площадками. Для измерения фототока использовалась техника синхронного детектирования сигнала с использованием усилителя SR830 c модуляцией излучения полупроводникового лазера Thorlabs L850P010 (длина волны 850 нм, непрерывный режим работы). Уровень токовой накачки лазера контролировался блоком управления Thorlabs LTC56B/M. Перед исследованием фототока графена на поверхности микрорезонатора была измерена мощность излучения лазера, падающая на образец, в зависимости от уровня токовой накачки лазера.

\section{3. Результаты и их обсуждение}

На рис. 1, $a$ представлено изображение диэлектрического микрорезонатора $\mathrm{SiO}_{2} / \mathrm{Ta}_{2} \mathrm{O}_{5}$ в геометрии поперечного сечения (110), полученное с помощью растровой электронной микроскопии (РЭМ) на микроскопе CamScan S4-90FE в режиме регистрации обратно отраженных электронов. Светлый контраст соответствует слоям $\mathrm{Ta}_{2} \mathrm{O}_{5}$, а темный - слоям $\mathrm{SiO}_{2}$. Следует отметить высокую планарность интерфейсов и однородность слоев по толщине диэлектрического микрорезонатора.

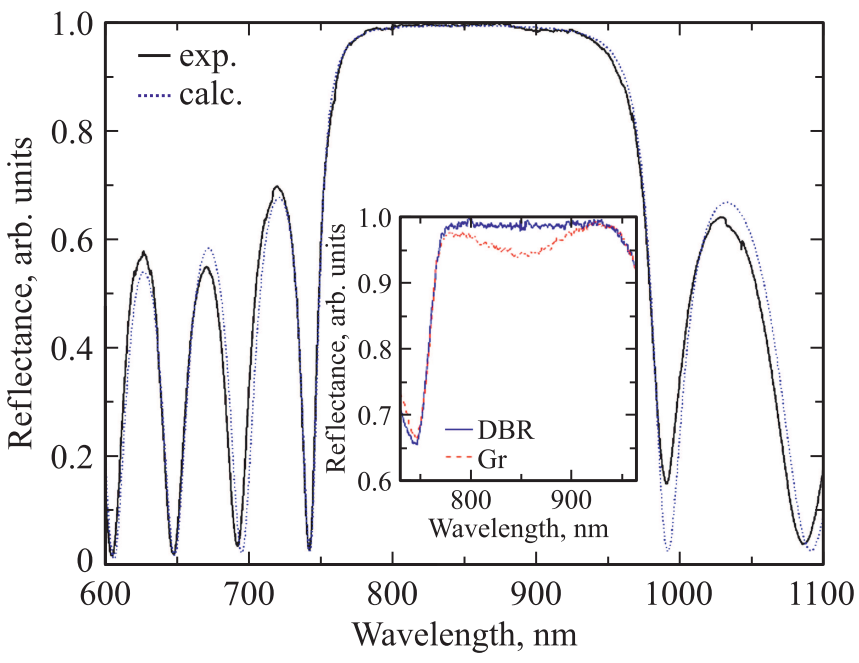

Рис. 2. Спектр оптического отражения диэлектрического резонатора (exp.) наряду с результатами моделирования (calc.). На вставке - спектры оптического отражения с пространственным разрешением $\sim 1$ мкм области микрорезонатора с графеном на его поверхности (Gr), диэлектрического микрорезонатора (DBR).

Для достижения высокой отражательной способности РБО необходимо уделить особое внимание шероховатости и оптической неоднородности используемых слоев. Для количественной оценки шероховатости микрорельефа поверхностей выполнялось двумерное преобразование Фурье АСМ-изображений топографии поверхности с последующим вычислением среднеквадратичное отклонения высот неровностей поверхности $R_{q}$ из интеграла функции спектральной плотности мощности поверхности твердого тела. Исследования показали, что наиболее развитую морфологию имеют слои аморфного $\mathrm{SiO}_{2}\left(R_{q} \approx 0.5-0.7 \mathrm{HM}\right)$, тогда как шероховатость слоев аморфного $\mathrm{Ta}_{2} \mathrm{O}_{5}$ лежит в пределах нескольких атомных ступеней полупроводниковой подложки $\left(R_{q} \approx 0.2-0.4 \mathrm{HM}\right)$. При увеличении количества осажденных слоев диэлектрика наблюдается некоторый рост шероховатости исследуемой поверхности, однако аморфный $\mathrm{Ta}_{2} \mathrm{O}_{5}$ оказывает планаризующий эффект и, как следствие, шероховатость поверхности диэлектрического микрорезонатора не превышает 1 нм.

На рис. 2 приведены экспериментальный и расчетный спектры оптического отражения диэлектрического микрорезонатора $\mathrm{SiO}_{2} / \mathrm{Ta}_{2} \mathrm{O}_{5}$. Поскольку спектры оптического отражения слоистых структур в первую очередь определяются оптическими толщинами слоев, то для адекватного моделирования в рамках метода матрицы переноса [53] были предварительно проведены исследования оптических параметров диэлектрических слоев $\mathrm{SiO}_{2}$ и $\mathrm{Ta}_{2} \mathrm{O}_{5}$ с помощью сканирующего спектрального фазово-модуляционного эллипсометра Horiba Uvisell 2. Затем с использованием модели дисперсии Тауца-Лоренца [54] были рассчитаны зависимости пока- 
зателя преломления и коэффициента экстинкции исследуемых материалов. Полоса высокого отражения задается нижним диэлектрическим РБО $\mathrm{SiO}_{2} / \mathrm{Ta}_{2} \mathrm{O}_{5}$, тогда как толщина оптического резонатора в основном определяет положение резонансной длины волны (в нашем случае резонанс не наблюдается ввиду отсутствия верхнего РБО и толщины резонатора, не кратной длине волны). Согласно экспериментальным данным, центр полосы отражения лежит вблизи 850-860 нм, а ширина полосы отражения по уровню 90\% достигает 200 нм, что хорошо коррелирует с результатами численного моделирования. Более того, наблюдается качественное согласие расчетных спектров оптического отражения с экспериментальными данными, что свидетельствует не только об однородности и воспроизводимости слоев по толщине, но и о корректности использованных оптических параметров. Таким образом, благодаря большому контрасту показателей преломления $\mathrm{SiO}_{2}-\mathrm{Ta}_{2} \mathrm{O}_{5}$ в совокупности с малой шероховатостью поверхности, высокой планарностью интерфейсов и сохранению периодичности РБО можно рассчитывать на реализацию отражательной способности нижнего РБО $\mathrm{SiO}_{2} / \mathrm{Ta}_{2} \mathrm{O}_{5}>99 \%$ в диапазоне длин волн 840-860 нм. Для оценки влияния слоя графена, помещенного в максимум электромагнитного поля оптической моды микрорезонатора, на отражательную способность диэлектрического микрорезонатора было проведено исследование спектров оптического отражения с пространственным разрешением $\sim 1$ мкм. На вставке к рис. 2 представлены спектры оптического отражения с пространственным разрешением $\sim 1$ мкм области диэлектрического микрорезонатора и области диэлектрического микрорезонатора с мезой в слое графена. Наблюдается появление локального минимума в полосе высокого отражения вблизи резонансной длины волны на кривой, соответствующей области мезы в слое графена (обозначение Gr) в сравнении с областью без графена (обозначение DBR), что обусловлено поглощением света в слое графена [25].

После формирования мез графена на поверхности диэлектрического микрорезонатора проведено измерение вольт-амперных характеристик (BAX) набора мез графена (свыше 200 мез графена, расположенных на 1/4 двухдюймовой пластины) с последующей оценкой методом АСМ шероховатости их поверхности с целью отбора для дальнейших исследований.

Поскольку при измерении ВАХ применялся двухзондовый метод, сопротивление $R$, полученное из анализа BAX, наряду с сопротивлением мезы в слое графена включает удвоенное контактное сопротивление, а также сопротивление токоподводящих проводов. Для оценки паразитного тока, протекающего по поверхности микрорезонатора, были измерены ВАХ мез без графена в аналогичной конфигурации контактов. Сопротивление данных мез превышало 20 МОм (при напряжении 5В), и, таким образом, подтверждается, что в ходе эксперимента в мезах с графеном исследовался транспорт носителей заряда именно графена, а не паразитный ток

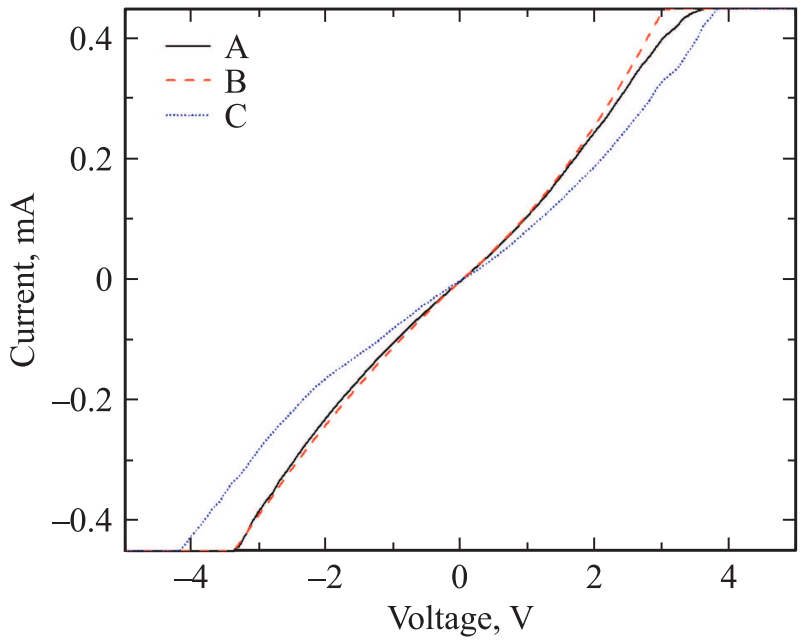

Рис. 3. Вольт-амперные характеристики мез А, В, С.

утечки по поверхности микрорезонатора. Из ВАХ мез графена следует слабое отклонение от линейности с ростом напряжения, а также разброс по сопротивлению (см. рис. 3). Среднее значение минимального сопротивления мез графена $R_{a v}$ составило $(10.4 \pm 3.0)$ кОм. Разброс по сопротивлению обусловлен вариацией площади мезы в слое графена ввиду недостаточной стойкости маски ФР к процессу травления. Стоит отметить, что слой графена между контактами визуально непрерывен во всех мезах. Для дальнейших исследований были выбраны 3 мезы в слое графена (А, В, С) с минимальным сопротивлением $(7.4,7.5,9.3$ кОм соответственно), отличающиеся геометрическими размерами. Проведена оценка шероховатости поверхности микрорезонатора в области мез с графеном. Среднеквадратическое отклонение высот неровностей поверхности $R_{q}$ составляет $\sim 3.8 \mathrm{Hм}$ в направлении сканирования параллельно контактным площадкам и $\sim 3.1$ нм в направлении протекания тока между контактными площадками, что может быть связано с доменной структурой поликристаллических пленок CVD-графена, имеющих выделенное направление [55]. Таким образом, шероховатость поверхности диэлектрического микрорезонатора с мезами в слое графена не превышает 4 нм.

Для оценки структурного качества мез в слое графена, сформированных на поверхности микрорезонатора, проведены исследования методом КРС. Спектры КРС были измерены в трех точках: по центру между контактами (точка „center"), слева от центра ( 1 мкм от левого контакта, точка „left“) и справа от центра ( 1 мкм от правого контакта, точка „right“). Исключение составила меза В, для которой спектры измерены в точке „сеntre“ и „right“. Спектр КРС, полученный после измерения фототока в точке „left", не удалось достоверно интерпретировать. Спектры КРС, соответствующие точке „center“, представлены на рис. 4, $a$. Все измеренные спектры КРС имеют особенности, характерные для 


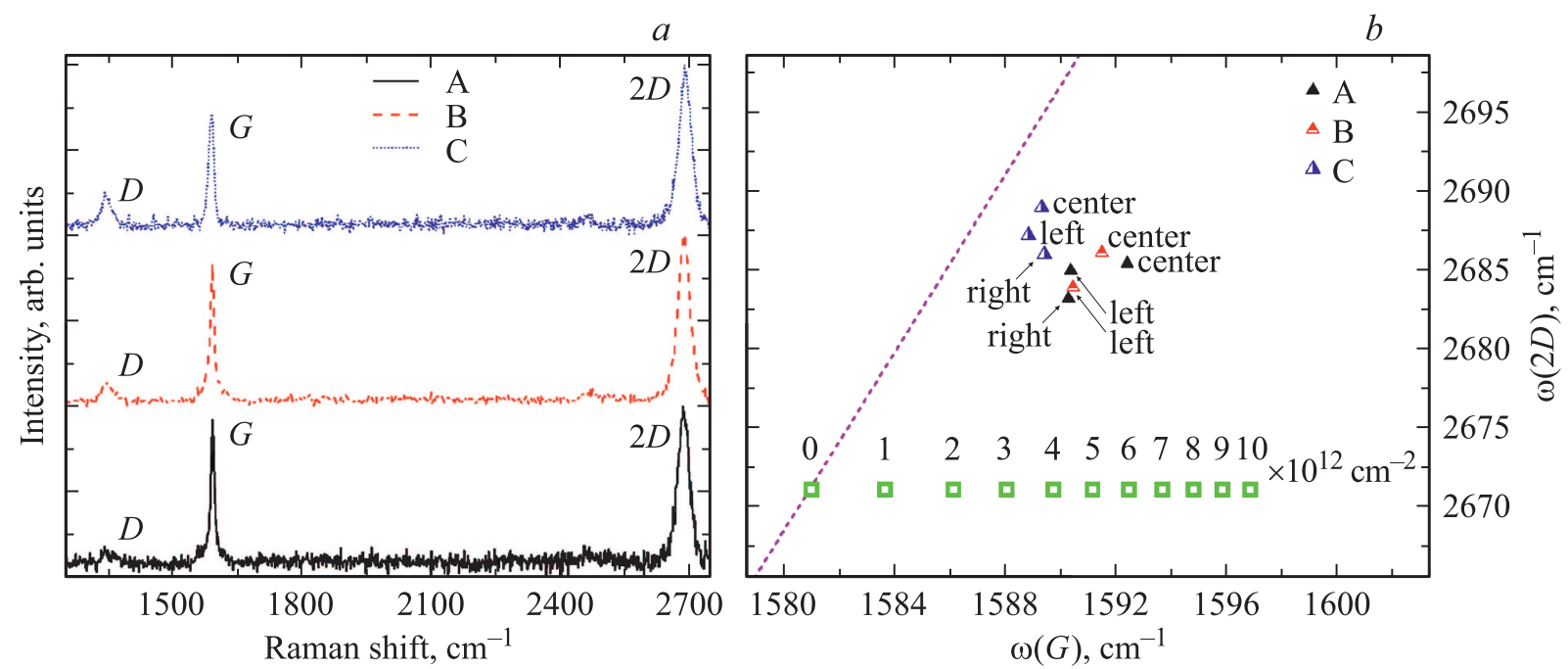

Рис. 4. $a$ - спектры КРС (нормированные по интенсивности линии $2 D$ ), измеренные в центральной точке (сеnter) по отношению к контактным областям. $b$ - данные о корреляции между положениями линий $G$ и $2 D$ (частоты $\omega(G), \omega(2 D)$ ) для трех различных мез; пунктирная линия - корреляция между положениями линий $G$ и $2 D$ в случае двуосной деформации [66], квадраты - зависимость сдвига линии $G$ от концентрации носителей заряда в случае нулевой деформации [67], треугольники экспериментальные данные; обозначения: center - измерения по центру между контактами, left и right - измерения слева $(\sim 1$ мкм от левого контакта) и справа от центра $(\sim 1$ мкм от правого контакта).

графена - линии $G$ (вблизи $1580 \mathrm{~cm}^{-1}$ ) и $2 D$ (вблизи $\left.2685 \mathrm{~cm}^{-1}\right),-$ а также линию $D$ (вблизи $1350 \mathrm{~cm}^{-1}$ ), появление которой свидетельствует о наличии дефектов в кристаллической решетке графена. Линия $2 D$ во всех спектрах КРС является симметричной, хорошо аппроксимируется контуром Лоренца и имеет достаточно малую ширину на полувысоте, FWHM, $<38 \mathrm{~cm}^{-1}$ (см. таблицу), что позволяет сделать вывод о монослойности исследуемого графена $[55,56]$. Границы зерен (кристаллитов) являются основным типом дефектов в случае графена, выращиваемого методом CVD [57]. Отношение интенсивностей линий $D$ и $G\left(I_{D} / I_{G}\right)$ позволяет оценить характерный размер зерен $L_{a}$ на основе выражения [58]: $L_{a}=(560) / \lambda^{4} \cdot\left(I_{D} / I_{G}\right)^{-1}$, где $\lambda-$ длина волны возбуждающего излучения. Результаты приведены в таблице. Средний размер зерен (кристаллитов) находится в диа- пазоне от 65 (меза С, точка „left“) до 177 нм (меза A, точка „сеnter").

В работе [59] представлен метод векторного разложения (vector-decomposition method), позволяющий разделить вклады концентрации носителей заряда и механических напряжений при анализе спектров КРС графена. На рис. $4, b$ представлены данные о корреляции между положениями линий $G$ и $2 D$ для трех исследованных мез графена (А, В, С). Результаты оценки величины двуосной деформации $\varepsilon_{\|}$и концентрации дырок $p$ с использованием метода векторного разложения представлены в таблице. Также в таблице представлены результаты оценки положения уровня Ферми $E_{\mathrm{F}}$, пересчитанного из значений $p$ по формуле [60] $E_{\mathrm{F}}=\hbar v_{\mathrm{F}} \sqrt{\pi|p|}$, где скорость Ферми для графена при расположении на подложке $\mathrm{SiO}_{2} v_{\mathrm{F}}=1.09 \cdot 10^{6} \mathrm{M} / \mathrm{c}[61,62], \hbar-$ постоянная Планка,

Параметры CVD-графена после формирования мез в слое графена и контактных площадок (обозначения приведены в тексте), полученные в результате анализа измеренных спектров КРС

\begin{tabular}{c|c|c|c|c|c|c|c|c}
\hline Меза & Точка & $\begin{array}{c}\text { FWHM }(D), \\
\mathrm{cm}^{-1}\end{array}$ & $\begin{array}{c}\text { FWHM }(G), \\
\mathrm{cm}^{-1}\end{array}$ & $\begin{array}{c}\text { FWHM }(2 D), \\
\mathrm{cm}^{-1}\end{array}$ & $\begin{array}{c}L_{a}, \\
\mathrm{HM}\end{array}$ & $\begin{array}{c}p, \\
10^{12} \mathrm{~cm}^{-2}\end{array}$ & $\begin{array}{c}E_{\mathrm{F}}, \\
\text { мэB B }\end{array}$ & $\begin{array}{c}\varepsilon_{\|}, \\
\%\end{array}$ \\
\hline \multirow{3}{*}{$\mathrm{A}$} & Right & 22 & 11 & 32 & 177 & 1.7 & 166 & -0.09 \\
& Center & 14 & 9 & 35 & 156 & 2.6 & 205 & -0.09 \\
& Left & 12 & 11 & 30 & 136 & 2.0 & 180 & -0.07 \\
\hline B & Right & 21 & 12 & 33 & 109 & 2.0 & 180 & -0.07 \\
& Center & 26 & 11 & 33 & 141 & 2.0 & 180 & -0.09 \\
\hline & Right & 25 & 16 & 37 & 98 & 0.9 & 107 & -0.09 \\
$\mathrm{C}$ & Center & 27 & 15 & 33 & 68 & 0.7 & 106 & -0.11 \\
& Left & 23 & 13 & 35 & 65 & 1.2 & 140 & -0.09
\end{tabular}




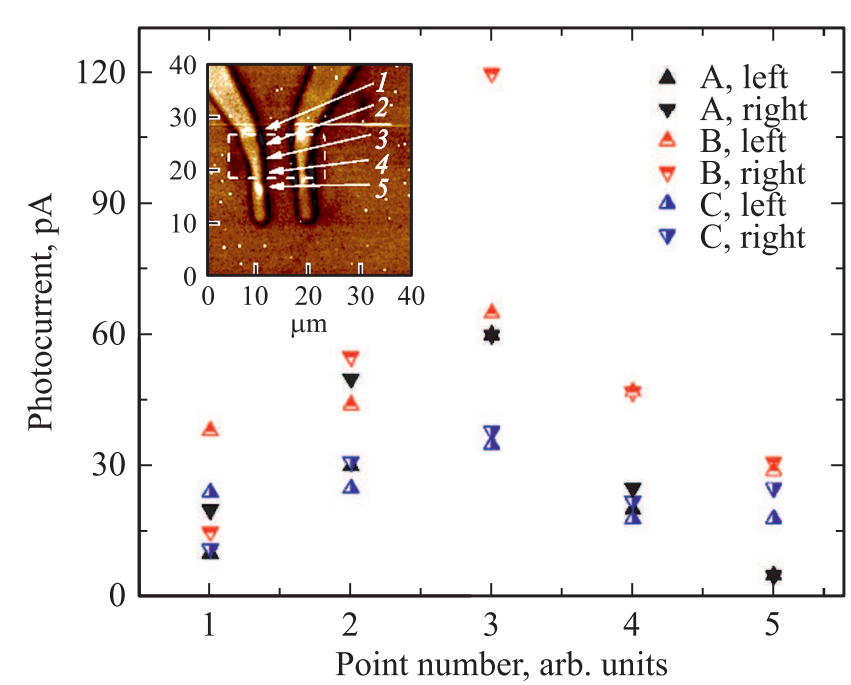

Рис. 5. Фототок, измеренный в различных точках образца для мез А, В, С. На оси абсцисс расположены номера областей, отмеченные на вставке рисунка. На вставке - визуализация распределения интенсивности отражения в спектральном диапазоне 630-960 нм; прямоугольная рамка на вставке соответствует области мезы в слое графена, вне области мезы графен вытравлен.

$\pi$ - число Пи. Полученные значения положения уровня Ферми согласуются с результатами, представленными в работе [55]. Максимальная концентрация носителей заряда соответствует центральной точке мезы А, причем вблизи контактных областей концентрация дырок падает. Для мезы В концентрация не зависит от области измерений. В свою очередь наименьшая концентрация носителей заряда как в центре мезы, так и в приконтактных областях наблюдалась на мезе С. Стоит также отметить, что концентрация дырок оказывается связанной с концентрацией дефектов. Так, на мезах А и В, в которых средний размер зерен (кристаллитов) составлял 157 и 125 нм соответственно, концентрация носителей заряда оказалась наибольшей, а наименьшие значения наблюдались на мезе $\mathrm{C}$, для которой средний размер зерен (кристаллитов) составил 77 нм. При этом графен в области С подвержен наиболее сильной деформации сжатия по сравнению с двумя другими областями.

В ходе эксперимента по измерению фототока для оптической накачки использовался лазер, излучение которого фокусировалось в пятно диаметром $\sim 1$ мкм, аналогично подходу, рассмотренному ранее [63]. Исследование амплитуды фототока, измеренного при мощности оптической накачки 9 мкВт, проведено в приконтактных областях (вблизи левого и правого контактов). На вставке к рис. 5 представлена визуализация распределения интенсивности отражения в спектральном диапазоне 630-960 нм. За пределами мезы графена (точки 1 и 5) величина фототока $I_{\mathrm{ph}}$ во всех мезах не превышает 20 пА. Максимум сигнала соответствует центру графеновой мезы в приконтактных областях (точке 3). Вблизи краев мез (точки 2,4) фототок падает по сравнению с фототоком, измеренным в позиции 3 при измерении как вблизи левого (обозначение „left“), так и вблизи правого (обозначение „right“) контактов. Максимальное значение $I_{\mathrm{ph}}$ составило 120 пА для мезы В (вблизи правого контакта, положение 3). Ранее для CVD-графена были получены значения фототока $\sim 50$ пА, однако измерения были проведены при температуре $20 \mathrm{~K}$ [63]. Согласно данным, представленным в таблице, для мезы А наблюдается снижение концентрации дырок при приближении к контакту. Снижение исходной концентрации дырок в приконтактных областях графена происходит вследствие компенсации электронами при использовании титана в качестве контактной металлизации $[26,64]$. Действительно, значение работы выхода титана составляет 4.3 эВ, что на 200 мэВ меньше работы выхода в графене [65]. Для мезы В в центральной части концентрация дырок ниже в сравнении с мезой А. Вблизи правого контакта концентрация дырок практически не изменяется, но уменьшается деформация сжатия. Вблизи правого контакта наблюдался максимальный фотоотклик, 120 пА. В данной точке в ходе анализа спектра КРС отмечались малый размер зерен (кристаллитов) и малая величина деформации сжатия. Более высокая концентрация дырок в сравнении с другими точками может приводить к более „резкому“ $p-n$-переходу [65-67] в сравнении с мезой А. В случае мезы С в приконтактных областях уровень легирования ниже, чем в мезах А, В, что приводит к снижению абсолютных значений фототока за счет „размытия“ области $p-n$-перехода.

\section{4. Заключение}

В ходе работы проведена отработка условий формирования методом реактивного магнетронного распыления диэлектрического РБО с высоким коэффициентом отражения. Диэлектрический РБО сформирован на основе пары материалов $\mathrm{SiO}_{2}$ и $\mathrm{Ta}_{2} \mathrm{O}_{5}$ для обеспечения высокой планарности гетерограниц РБО и низкой шероховатости поверхности диэлектрического микрорезонатора. Проведена оптимизация условий переноса и формирования мез на основе CVD-графена. Диагностика структурного качества методом КРС свидетельствует о монослойности CVD-графена и наличии небольшого количества дефектов, связанных с границами зерен (кристаллитов). На основе анализа спектров КРС выполнена оценка концентрации дырок, а также величины двуосной деформации графена в центральной и приконтактных областях. Проведено измерение величины фототока при локальной оптической накачке. Максимальный фотоотклик, измеренный при комнатной температуре, составил $\sim 120$ пА. Дальнейшие шаги исследований связаны с отработкой технологии формирования верхнего диэлектрического РБО на поверхности мезы графена и созданием резонансного фотодетектора на его основе. 


\section{Финансирование работы}

Работа выполнена при финансовой поддержке гранта Российского научного фонда (проект № 18-72-00157).

\section{Конфликт интересов}

Авторы заявляют, что у них нет конфликта интересов.

\section{Список литературы}

[1] Q. Yu, Y. Wang, L. Xie, S. Nadri, K. Sun, J. Zang, Q. Li, R.M. Weikle, A. Beling. OSA Technical Digest, SM2I.1 (2018). DOI: 10.1364/CLEO_SI.2018.SM2I.1

[2] A.M. Joshi, S. Datta. Proc. SPIE, 10528, 105280 Y (2018). DOI: $10.1117 / 12.2292979$

[3] K.J. Tielrooij, L. Piatkowski, M. Massicotte, A. Woessner, Q. Ma, Y. Lee, K.S. Myhro, C.N. Lau, P. Jarillo-Herrero, N.F. van Hulst, F.H.L. Koppens. Nature Nanotechnol., 10 (5), 437 (2015).

[4] S. Schuler, D. Schall, D. Neumaier, L. Dobusch, O. Bethge, B. Schwarz, M. Krall, T. Mueller. Nano Lett., 16 (11), 7107 (2016).

[5] A. Bablich, S. Kataria, M. Lemme. Electronics, 5 (4), 13 (2016).

[6] D. Schall, C. Porschatis, M. Otto, D. Neumaier. J. Phys. D: Appl. Phys., 50 (12), 124004 (2017).

[7] Z. Ni, L. Ma, S. Du, Y. Xu, M. Yuan, H. Fang, Z. Wang, M. Xu, D. Li, J. Yang, W. Hu, X. Pi, D. Yang. ACS Nano, 11 (10), 9854 (2017).

[8] J. Fang, D. Wang, C.T. DeVault, T.-F. Chung, Y.P. Chen, A. Boltasseva, V.M. Shalaev, A.V. Kildishev. Nano Lett., 17 (1), 57 (2016).

[9] M. Romagnoli, V. Sorianello, M. Midrio, F.H.L. Koppens, C. Huyghebaert, D. Neumaier, P. Galli, W. Templ, A. D’Errico, A.C. Ferrari. Nature Rev. Mater., 3 (10), 392 (2018).

[10] G. Wang, M. Zhang, D. Chen, Q. Guo, X. Feng, T. Niu, X. Liu, A. Li, J. Lai, D. Sun, Z. Liao, Y. Wang, P.K. Chu, G. Ding, X. Xie, Z. Di, X. Wang. Nature Commun., 9 (1), 5168 (2018).

[11] V. Shautsova, T. Sidiropoulos, X. Xiao, N.A. Güsken, N.C.G. Black, A.M. Gilbertson, V. Giannini, S.A. Maier, L.F. Cohen, R.F. Oulton. Nature Commun., 9(1), 5190 (2018).

[12] S. Schuler, D. Schall, D. Neumaier, B. Schwarz, K. Watanabe, T. Taniguchi, T. Mueller. ACS Photonics, 5 (12), 4758 (2018).

[13] T.J. Yoo, Y.J. Kim, S.K. Lee, C.G. Kang, K.E. Chang, H.J. Hwang, N. Revannath, B.H. Lee. ACS Photonics, 5 (2), 365 (2017).

[14] Y. Zhang, H. Zheng, Q. Wang, C. Cong, L. Hu, P. Tian, R. Liu, S.-L. Zhang, Z.-J. Qiu. Small, 14 (24), 1800691 (2018).

[15] H. Suzuki, N. Ogura, T. Kaneko, T. Kato. Sci. Rep., 8(1), 11819 (2018).

[16] Q. Ma, C.H. Lui, J.C.W. Song, Y. Lin, J. F. Kong, Y. Cao, T.H. Dinh, N.L. Nair, W. Fang, K. Watanabe, T. Taniguchi, S.-Y. Xu, J. Kong, T. Palacios, N. Gedik, N.M. Gabor, P. Jarillo-Herrero. Nature Nanotechnol., 14 (2), 145 (2018).

[17] M. Long, P. Wang, H. Fang, W. Hu. Adv. Func. Mater., 29 (19), 1803807 (2018).
[18] A. Blaikie, D. Miller, B.J. Alemán. Nature Commun., 10 (1), 4726 (2019).

[19] V. Kilic, M.A. Foster, J.B. Khurgin. Appl. Phys. Lett., 115 (16), 161106 (2019).

[20] J.E. Muench, A. Ruocco, M.A. Giambra, V. Miseikis, D. Zhang, J. Wang, H.F.Y. Watson, G.C. Park, S. Akhavan, V. Sorianello, M. Midrio, A. Tomadin, C. Coletti, M. Romagnoli, A.C. Ferrari, I. Goykhman. Nano Lett., 19(11), 7632 (2019).

[21] S. Marconi, V. Miseikis, M.A. Giambra, A. Montanaro, V. Sorianello, B. Torres, I. Goykhman, C. Coletti, F. Koppens, A.C. Ferrari, M. Romagnoli. OSA Technical Digest (San Jose, USA, 2019) p. STh4N.2. DOI: 10.1364/CLEO_SI.2019.STh4N.2

[22] P. Huang, E. Riccardi, S. Messelot, H. Graef, F. Valmorra, J. Tignon, T. Taniguchi, K. Watanabe, S. Dhillon, B. Plaçais, R. Ferreira, J. Mangeney. Nature Commun., 11 (1), 863 (2020).

[23] Y. Ding, Z. Cheng, X. Zhu, K. Yvind, J. Dong, M. Galili, H. Hu, N.A. Mortensen, S. Xiao, L.K. Oxenløwe. Nanophotonics, 9 (2), 317 (2020).

[24] E.D. Palik. Handbook of Optical Constants of Solids (N. Y., USA, Academic Press, 1985).

[25] R.R. Nair, P. Blake, A.N. Grigorenko, K.S. Novoselov, T.J. Booth, T. Stauber, N.M.R. Peres, A.K. Geim. Science, 320 (5881), 1308 (2008).

[26] F.H.L. Koppens, T. Mueller, P. Avouris, A.C. Ferrari, M.S. Vitiello, M. Polini. Nature Nanotechnol., 9(10), 780 (2014).

[27] K.F. Mak, L. Ju, F. Wang, T.F. Heinz. Solid State Commun., 152 (15), 1341 (2012).

[28] A.B. Kuzmenko, E. van Heumen, F. Carbone, D. van der Marel. Phys. Rev. Lett., 100 (11), 117401 (2008).

[29] T.J. Echtermeyer, L. Britnell, P.K. Jasnos, A. Lombardo, R.V. Gorbachev, A.N. Grigorenko, A.K. Geim, A.C. Ferrari, K.S. Novoselov. Nature Commun., 2 (1), 458 (2011).

[30] Y. Liu, R. Cheng, L. Liao, H. Zhou, J. Bai, G. Liu, L. Liu, Y. Huang, X. Duan. Nature Commun., 2 (1), 579 (2011).

[31] A. Pospischil, M. Humer, M.M. Furchi, D. Bachmann, R. Guider, T. Fromherz, T. Mueller. Nature Photonics, 7 (11), 892 (2013).

[32] D. Schall, C. Porschatis, M. Otto, D. Neumaier. J. Phys. D: Appl. Phys., 50 (12), 124004 (2017).

[33] S. Thongrattanasiri, F.H.L. Koppens, F.J. García de Abajo. Phys. Rev. Lett., 108 (4), 047401 (2012).

[34] H. Yan, X. Li, B. Chandra, G. Tulevski, Y. Wu, M. Freitag, W. Zhu, P. Avouris, F. Xia, Nature Nanotechnol., 7 (5), 330 (2012).

[35] G. Konstantatos, M. Badioli, L. Gaudreau, J. Osmond, M. Bernechea, F.P.G. de Arquer, F. Gatti, F.H.L. Koppens. Nature Nanotechnol., 7 (6), 363 (2012).

[36] M. Engel, M. Steiner, A. Lombardo, A.C. Ferrari, H.V. Löhneysen, P. Avouris, R. Krupke. Naure Commun., 3 (1), 906 (2012).

[37] M. Casalino, U. Sassi, I. Goykhman, A. Eiden, E. Lidorikis, S. Milana, D. De Fazio, F. Tomarchio, M. Iodice, G. Coppola, A.C. Ferrari. ACS Nano, 11 (11), 10955 (2017).

[38] M. Casalino. J. Lightwave Technol., 36 (9), 1766 (2018).

[39] B. Vasić, R. Gajić. Optics Lett., 39 (21), 6253 (2014).

[40] T. Mueller, M. Furchi, A. Urich, A. Pospischil. Proc. SPIE, 8600, 86001H (2013). DOI: $10.1117 / 12.2001886$ 
[41] S.A. Blokhin, M.A. Bobrov, A.G. Kuzmenkov, A.A. Blokhin, A.P. Vasil'ev, Y.A. Guseva, M.M. Kulagina, I.O. Karpovsky, Y.M. Zadiranov, S.I. Troshkov, N.D. Prasolov, P.N. Brunkov, V.S. Levitsky, V. Lisak, N.A. Maleev, V.M. Ustinov. Techn. Phys. Lett., 42 (10), 1049 (2016).

[42] N.V. Kryzhanovskaya, E.I. Moiseev, A.G. Gladyshev, L.Y. Karachinsky, I.I. Novikov, A.V. Babichev, S.A. Blokhin, M.A. Bobrov, Y.M. Zadiranov, S.I. Troshkov, A.Y. Egorov. Proc. SPIE, 10098, 1009811 (2017).

[43] A.V. Babichev, H. Zhang, P. Lavenus, F.H. Julien, A.Y. Egorov, Y.T. Lin, L.W. Tu, M. Tchernycheva. Appl. Phys. Lett., 103 (20), 201103 (2013).

[44] L. Mancini, M. Morassi, C. Sinito, O. Brandt, L. Geelhaar, H.-G. Song, Y.-H. Cho, N. Guan, A. Cavanna, J. Njeim, A. Madouri, C. Barbier, L. Largeau, A. Babichev, F.H. Julien, L. Travers, F. Oehler, N. Gogneau, J.-C. Harmand, M. Tchernycheva. Nanotechnology, 30 (21), 214005 (2019).

[45] V. Kumaresan, L. Largeau, A. Madouri, F. Glas, H. Zhang, F. Oehler, A. Cavanna, A. Babichev, L. Travers, N. Gogneau, M. Tchernycheva, J.-C. Harmand. Nano Lett., 16 (8), 4895 (2016).

[46] A.V. Babichev, D.V. Denisov, M. Tchernycheva, F.H. Julien, H. Zhang. Techn. Phys. Lett., 44 (12), 1111 (2018).

[47] A.V. Babichev, V.E. Gasumyants, A.Y. Egorov, S. Vitusevich, M. Tchernycheva. Nanotechnology, 25 (33), 335707 (2014).

[48] A.V. Babichev, V.Y. Butko, M.S. Sobolev, E.V. Nikitina, N.V. Kryzhanovskaya, A.Y. Egorov. Semiconductors, 46 (6), 796 (2012).

[49] M. Morassi, N. Guan, V.G. Dubrovskii, Y. Berdnikov, C. Barbier, L. Mancini, L. Largeau, A.V. Babichev, V. Kumaresan, F.H. Julien, L. Travers, N. Gogneau, J.-C. Harmand, M. Tchernycheva. Cryst. Growth Des., 20 (2), 552 (2020).

[50] M. Tchernycheva, P. Lavenus, H. Zhang, A.V. Babichev, G. Jacopin, M. Shahmohammadi, F.H. Julien, R. Ciechonski, G. Vescovi, O. Kryliouk. Nano Lett., 14 (5), 2456 (2014).

[51] A.V. Babichev, V.E. Gasumyants, V.Y. Butko. J. Appl. Phys., 113 (7), 076101 (2013).

[52] A.V. Babichev, S.A. Rykov, M. Tchernycheva, A.N. Smirnov, V.Y. Davydov, Y.A. Kumzerov, V.Y. Butko. ACS Appl. Mater. Interfaces, 8 (1), 240 (2015).

[53] М. Борн, Э. Вольф. Основы оптики (М., Наука, 1970).

[54] G.E. Jellison, F.A. Modine. Appl. Phys. Lett., 69 (3), 371 (1996).

[55] D. De Fazio, D.G. Purdie, A.K. Ott, P. Braeuninger-Weimer, T. Khodkov, S. Goossens, T. Taniguchi, K. Watanabe, P. Livreri, F.H.L. Koppens, S. Hofmann, I. Goykhman, A.C. Ferrari, A. Lombardo. ACS Nano, 13 (8), 8926 (2019).

[56] A.C. Ferrari, J.C. Meyer, V. Scardaci, C. Casiraghi, M. Lazzeri, F. Mauri, S. Piscanec, D. Jiang, K.S. Novoselov, S. Roth, A.K. Geim. Phys. Rev. Lett., 97 (18), 187401 (2006).

[57] L. Colombo, X. Li, B. Han, C. Magnuson, W. Cai, Y. Zhu, R.S. Ruoff. ECS Trans., 28, 109 (2010).

[58] L.G. Cançado, K. Takai, T. Enoki, M. Endo, Y.A. Kim, H. Mizusaki, A. Jorio, L.N. Coelho, R. Magalhães-Paniago, M.A. Pimenta. Appl. Phys. Lett., 88 (16), 163106 (2006).

[59] J.E. Lee, G. Ahn, J. Shim, Y.S. Lee, S. Ryu. Nature Commun., 3 (1), 1024 (2012)

[60] C.-F. Chen, C.-H. Park, B.W. Boudouris, J. Horng, B. Geng, C. Girit, A. Zettl, M.F. Crommie, R.A. Segalman, S.G. Louie, F. Wang. Nature, 471 (7340), 617 (2011).
[61] K.R. Knox, S. Wang, A. Morgante, D. Cvetko, A. Locatelli, T.O. Mentes, M.A. Niño, P. Kim, R.M. Osgood. Phys. Rev. B, 78 (20), 201408 (2008).

[62] R.S. Deacon, K.-C. Chuang, R.J. Nicholas, K.S. Novoselov, A.K. Geim. Phys. Rev. B, 76 (8), 081406 (2007).

[63] D. Sun, G. Aivazian, A.M. Jones, J.S. Ross, W. Yao, D. Cobden, X. Xu. Nature Nanotechnol., 7 (2), 114 (2012).

[64] G. Giovannetti, P.A. Khomyakov, G. Brocks, V.M. Karpan, J. van den Brink, P.J. Kelly. Phys. Rev. Lett., 101 (2), 026803 (2008).

[65] E.J.H. Lee, K. Balasubramanian, R.T. Weitz, M. Burghard, K. Kern. Nature Nanotechnol., 3 (8), 486 (2008).

[66] F. Ding, H. Ji, Y. Chen, A. Herklotz, K. Doörr, Y. Mei, A. Rastelli, O.G. Schmidt. Nano Lett., 10 (9), 3453 (2010).

[67] G. Froehlicher, S. Berciaud. Phys. Rev. B, 91 (20), 205413 (2015).

Редактор Л.В. Шаронова

\section{A research of the photoresponse of graphene produced by chemical vapor deposition}

A.V. Babichev' ${ }^{1}$, S.A. Kadinskaya ${ }^{1}$, K.Yu. Shubina ${ }^{1}$,

A.A. Vasiliev' ${ }^{1}$, A.A. Blokhin',3, E.I. Moiseev' ${ }^{4}$,

S.A. Blokhin ${ }^{3}$, I.S. Mukhin ${ }^{1,5}$, I.A. Eliseyev ${ }^{3}$,

V.Yu. Davydov ${ }^{3}$, P.N. Brunkov ${ }^{3}$,

N.V. Kryzhanovskaya ${ }^{4}$, A.Yu. Egorov ${ }^{5}$

${ }^{1}$ St. Petersburg National Research Academic University, Russian Academy of Sciences,

194021 St. Petersburg, Russia

${ }^{2}$ Submicron Heterostructures for Microelectronics,

Research \& Engineering center,

Russian Academy of Sciences,

194021 St. Petersburg, Russia

${ }^{3}$ loffe Institute,

194021 St. Petersburg, Russia

${ }^{4}$ National Research University

Higher School of Economics,

190008 St. Petersburg, Russia

${ }^{5}$ ITMO University,

197101 St. Petersburg, Russia

Abstract The paper presents the results of experiments in the fabrication and research of properties of photodetector structures on the basis of monolayer graphene produced by chemical vapor deposition. The base structure was the geometry of a $\mathrm{Ta}_{2} \mathrm{O}_{5}$ vertical microcavity with a lower dielectric $\mathrm{SiO}_{2} / \mathrm{Ta}_{2} \mathrm{O}_{5}$ distributed Bragg reflector with a resonance wavelength of about $850 \mathrm{~nm}$. The conditions were optimized for the transfer and fabrication of mesas in the graphene layer on the microcavity surface. The diagnostics of the structural quality of graphene after the fabrication of mesas in the graphene layer and contact pads by Raman spectroscopy evidence the monolayer structure of graphene with a low singularity strength in its spectrum that is responsible for the structure imperfection. The photocurrent value at local optical pumping was measured. 